\title{
Erratum to: Classification of periodontal biotypes with the use of CBCT. A cross-sectional study
}

\author{
Maria Nikiforidou ${ }^{1}$ - Lazaros Tsalikis ${ }^{1}$ - Christos Angelopoulos ${ }^{2}$. \\ Georgios Menexes $^{3} \cdot$ Ioannis Vouros ${ }^{1} \cdot$ Antonios Konstantinidis $^{1}$
}

Published online: 18 June 2016

(C) Springer-Verlag Berlin Heidelberg 2016

Erratum to: Clinical Oral Investigations

DOI 10.1007/s00784-015-1694-y

The names in the original version of this article were inversed.

Correct names are presented here.

The online version of the original article can be found at http://dx.doi. org/10.1007/s00784-015-1694-y.

\section{Maria Nikiforidou}

mnikifor@yahoo.gr

1 Department of Preventive Dentistry, Periodontology and Implant Biology, School of Dentistry, Aristotle University of Thessaloniki, Agiou Dimitriou 195, 54124 Thessaloniki, Greece

2 Department of Oral Surgery, Implantology and Roentgenology, School of Dentistry, Aristotle University of Thessaloniki, Agiou Dimitriou 195, 54124 Thessaloniki, Greece

3 School of Agriculture, Laboratory of Agronomy, Aristotle University of Thessaloniki, Agiou Dimitriou 195, 54124 Thessaloniki, Greece 